\title{
'Red Ruby': an interactive web-based intervention for lifestyle modification on metabolic syndrome: a study protocol for a randomized controlled trial
}

\author{
Leila Jahangiry', Davoud Shojaeizadeh ${ }^{2}$, Mahdi Najafi ${ }^{3}$, Kazem Mohammad $^{4}$, Mahdieh Abbasalizad Farhangi ${ }^{5}$ \\ and Ali Montazeri ${ }^{*}$
}

\begin{abstract}
Background: Although effectiveness of web-based interventions on lifestyle changes are recognized, the potential of such programs on metabolic syndrome has not been explored. We describe the protocol of a randomized controlled trial that aims to determine the feasibility, acceptability, usability, and effectiveness of interactive technology on lifestyle intervention in a population with metabolic syndrome.

Methods/design: This is a two-arm randomized controlled trial. The study includes 160 participants $(n=80$ per arm) who will be recruited via online registration on the study website. The inclusion criteria are that they should have metabolic syndrome and have access to the Internet. All participants will receive information on dietary intake and physical activity through the study website. The intervention group will receive additional resources via the study website including interactive Healthy Heart Profile and calorie restricted diet tailored to the participants. The primary outcomes are feasibility, acceptability, usability, and the change in metabolic syndrome components. The secondary outcomes are comparing quality of life, physical activity and food intake among the study arms. The participants will be followed up to 6 months with data collection scheduled at baseline, 3 and 6 months.
\end{abstract}

Discussion: There is a need for developing and evaluating web-based interventions that target people with high risk for cardiovascular diseases. This study will therefore make an important contribution to this novel field of research and practice.

Trial registration: $I R C T 201111198132 \mathrm{~N} 1$

\section{Background}

Cardiovascular diseases (CVD) are the leading cause of mortality and morbidity worldwide and a growing health problem among developing nations [1,2]. It is argued that metabolic syndrome is the most responsible risk factor for developing the disease [3-6]. The National Cholesterol Education Program Adult Treatment Panel (ATP) III defined metabolic syndrome as the presence of three or more of the following conditions: triglyceride level of at least $150 \mathrm{mg} / \mathrm{dl}$, HDL level less than $40 \mathrm{mg} / \mathrm{dl}$ in men and less than $50 \mathrm{mg} / \mathrm{dl}$ in women, systolic/diastolic blood

\footnotetext{
* Correspondence: Shojae5@yahoo.com; montazeri@acecr.ac.ir ${ }^{2}$ Health Education and Health Promotion Department, School of Public Health, Tehran University of Medical Sciences, Tehran, Iran

${ }^{6}$ Mental Health Research Group, Health Metrics Research Center, Iranian Institutes for Health Sciences Research, ACECR, Tehran, Iran

Full list of author information is available at the end of the article
}

pressure 130/85 mm $\mathrm{Hg}$ or higher, fasting blood glucose level $110 \mathrm{mg} / \mathrm{dl}$ or higher and waist circumference greater than $102 \mathrm{~cm}$ in men and greater than $88 \mathrm{~cm}$ in women [7]. Metabolic syndrome is affecting about $20 \%$ of adult population without known diabetes and cardiovascular diseases and is associated with increasing cardiovascular morbidity and mortality [8,9]. Ischemic heart disease was the leading cause of Disability-Adjusted Life Years (DALYs) worldwide in 2010 [10].

Recent evidence indicates that individuals with metabolic syndrome are approximately twice more likely to develop cardiovascular disease and between 3.5 and 5 times more likely to develop type 2 diabetes [11]. The prevalence of metabolic syndrome in Iran is very high. The Tehran Lipid and Glucose study (TLGS) reported that $42 \%$ of women and $24 \%$ of men were suffering from metabolic syndrome. Metabolic syndrome has been

\section{() Biomed Central}


identified by central obesity, increased triglycerides, reduced high-density lipoprotein cholesterol (HDL), hypertension, and elevated fasting blood glucose concentration [12-14]. Increased cardiovascular mortality and metabolic syndrome morbidity in developing countries is largely due to the changes in lifestyle including changes in dietary habits and lack of exercise [15-17]. Therefore effective and affordable strategies to control the syndrome would benefit the at risk population.

In the past there have been several different strategies to fight metabolic syndrome. Although traditional lifestyle interventions such as face-to-face diet and exercise programs have succeeded in treating the metabolic syndrome $[18,19]$, clinical practice and research have shown significant difficulties with regard to availability, cost, treatment adherence and long-term efficacy of these procedures [20]. Indeed issues such as time and travel demands typically were identified as problems with traditional programs [21]. However, the new communication technologies provided alternatives that are becoming very popular. For instance the Internet penetration rapidly is growing and its use in medicine and health care are ever increasing. In particular web-based interventions has attracted a considerable attention as an approach in medical discipline.

A web-based intervention provides easily access to evidence-based content in every time and makes it feasible to increase tremendously the number of people reached to information [20,22,23]. According to Barak et al. a web-based intervention is: 'a primarily self-guided intervention program that is executed by means of a prescriptive online program operated through a website and used by consumers seeking health- and mental-health related assistance' [24]. The effectiveness of web-based interventions has been demonstrated for numerous health behaviors and chronic conditions. Review studies of webbased interventions reported positive results for smoking cessations [25-31], substance abuse [32], alcohol consumption [28,33-39], weight management [20,40-46], diabetes care $[47,48]$, depression, anxiety and stress [49-55], physical activity and nutrition [56-61] and chronic conditions $[62,63]$. However, the effectiveness of these online applications is limited by high attrition rates and the fact that only few users visit a health intervention website more than once $[64,65]$. In addition, it is argued although there is some evidence for the effectiveness of web-based interventions for improving healthy behaviors, it is clear that maintaining improvements remain a challenging issue [66].

There is evidence that among the web-based interventions for lifestyle changes those that benefit from interactive technology are more effective than those that provide one-way communications [66]. Interactive webbased technology is a two-way communication by which using the Internet, people could interact with provider. Interactive web-based technologies have several advantages. Access to credible information is quick and easy to read updated information [66]. Lower cost, better interaction, less time between screening and feedback are other returns of interactive web-based interventions $[59,67]$. Interactive web-based programs can offer tailored information and messages to participants to personalize their experience and apply interventions in the privacy of their home and at the convenient time [23]. In fact by using interactive web-based technology one could design web-based interventions tailored to the need of target population. Therefore with the growth of new and exciting interactive technologies, opportunities to use this novel applicable approach is recommended to design easy to use, engaging, continual self-monitoring program for metabolic syndrome.

Internet penetration in Iran is $53.3 \%$ and $46.7 \%$ of people are using the Internet [68]. A recent study indicated that $32.7 \%$ of people living in Tehran (the capital) have used Internet at the past 12 months [69]. As any other communities the use of the Internet in Iran is rapidly increasing. However, in spite of that increase, we are not aware of any evaluation of web-based health programs conducted in Tehran, Iran.

\section{Aim}

The aim of this randomized controlled trial (RCT) is to determine the feasibility, acceptability, usability, and effectiveness of an interactive web-based lifestyle intervention focusing on dietary and physical activity for reducing metabolic syndrome indicators. It was hypothesized that developing and launching an interactive web-based educational program would be feasible, acceptable and will be used by target audiences, and that using interactive web-based educational program will produce a change in lifestyle behaviors and this consequently will lead to reduction in metabolic syndrome.

\section{Methods}

\section{Trial design}

This study is an interactive web-based randomized trial (using pre and post-intervention assessments). Participants in this parallel study randomly will be assigned to one of two conditions: the intervention group and the waiting list group (the control group). Participants will be assessed at three points in time: at baseline, three and six months follow-up. Study procedure from enrollment through follow up data collection and analysis are depicted in Figure 1.

\section{Study procedure}

An interactive web page namely Healthy Heart Education (http://www.Heartresearch.ir) is designed in order to 


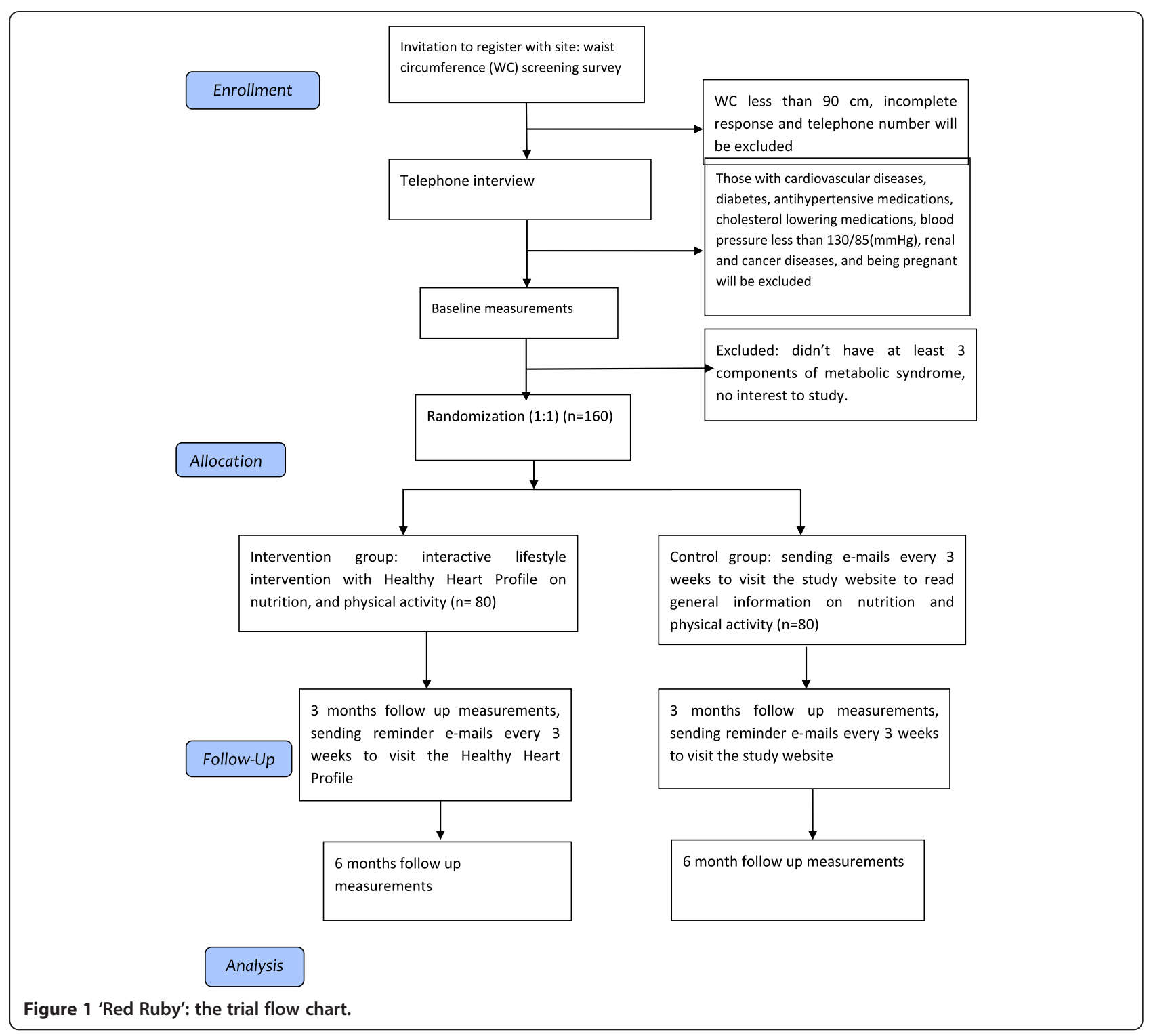

increase awareness of visitors about decreasing cardiovascular diseases and attract the attention of users for metabolic syndrome [Figure 2]. It is a free website providing information and advice about reducing heart diseases. This public website presents general information about hypertension, metabolic syndrome, diabetes, obesity and central obesity, nutrition for healthy heart and cardiovascular diseases (types, symptoms, prevention). The general educational materials are available in HTML and PDF formats. There is a registration page that invites people to take part in a study named the 'Red Ruby'. The registration page includes recording of information on name, gender, age, waist circumference, weight, e-mail and address. The homepage also shows how to measure waist circumference.

\section{Recruitment}

The study will be advertised by posters in virtual and non-virtual environments by announcing the web site address and indicating that people could visit the site for further information. Then those who visit the site will be invited to participate in the study by registering in the site. Accordingly the website's database will be reviewed by a trained research assistance in order to identify registrant aged 20 and over living in Tehran (the study setting). Then, those who meet the study criteria will be contacted by the main investigator (LJ). Finally identified eligible participants who interested in pursuing the study, will ask to schedule for a free clinic visit and clinical measurements by a trained nursing staff at Tehran Heart Center. 


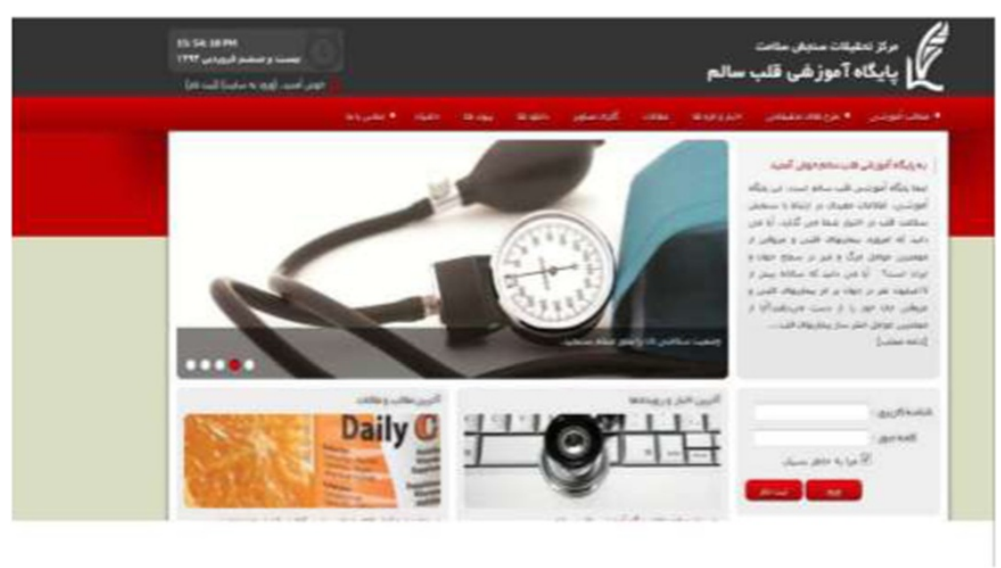

Figure 2 A snapshot of the website.

\section{Participants}

Participants are individuals that should have metabolic syndrome aged 20 years and above living in Tehran registered on the study website.

\section{Inclusion criteria}

a) Waist circumference $\geq 90$ (cut-off for metabolic syndrome in Iran for both gender [70-72]), b) Blood pressure $\geq 130 / 85$, c) Access to Internet, d) Having simple skills to work with Internet

\section{Exclusion criteria}

a) Having history of cardiovascular diseases, b) Diabetics, c) Having cancer, d) Patients with renal diseases, e) Being pregnant, f) Taking medication for hypertension, g) Taking medication for dislipidemia, and h) Having incomplete registration form

\section{Randomization}

Randomization will be carried out after baseline measurements. Participants will be randomly assigned to the trial arms. Randomization sequence will created manually by a biostatistician using Excel software [Command in the Excel for random block sizes column: =rand()] [73] to assign participants to the study arms using a 1:1 allocation ratio with block size of 4 . The allocation sequence will conceal from the investigators in sequentially numbered, opaque, sealed and stapled envelopes.

\section{Intervention}

Both intervention and control arms will be encouraged to engage in making healthy changes to their physical activity and nutrition behaviors in order to treat metabolic syndrome. The participants in intervention arm will be received the username and password to use the My Healthy Heart Profile and log-in to the interactive section of website. Participants in intervention arm will be encouraged to frequently log-in and visit their homepage or ask questions at any time they wish. For the security and confidentiality, the users will receive personal username and password. We will recommend the participants to keep the password safe and avoid sharing it with anyone. They will not able to change the password. If any one forgets the password a new one will be sent to his/her e-mails address.

\section{My healthy heart profile}

My Healthy Heart Profile is an interactive web-based program designed for reducing metabolic syndrome. The program is developed according to the feedback and monitoring' as a behavior change technique [74]. It is designed to send feedback to each participant according to his/her metabolic syndrome indicators. In fact the participants will receive text-based messages and graphic feedbacks immediately after compellation of each records of risk factors.

My Healthy Heart Profile is structured in six parts and includes:

1. Personal home page: on the main page educational materials will be uploaded every 2 weeks. The titles are: Do you know your cholesterol level?, Control your high blood pressure, Are you at risk for heart disease, Do you need to lose weight?, Protect your heart against diabetes, Physical activity and your heart, and Lowering blood pressure. Users can download and read all educational materials or save and print to read it at convenient time.

2. Personal information includes name, age, gender, weight, height, telephone number, and e-mail address.

3. Inbox as an interactive section in the profile for personal questions. Participants in intervention arm can send personal questions and receive answers. 
We will send a calorie-restricted tailored diet to all participants' inbox provided by a dietitian. The calorie restricted diet will be based on each participant's calorie requirement according to his/ her ideal body weight (IBW) and corrected body weight (CBW) with less than $30 \%$ of calories derived from fat, in accordance with the National Heart, Lung, and Blood Institute guidelines [75]. IBW will be calculated with Hamwi equation [76]. CBW is defined as ((IBW + 25\% (actual body weight IBW)). We will request from intervention group to adhere the dieting program.

4. A cardiovascular risk estimation tool for 10-years (based on the Persian online version of Framingham Risk Score-FSR). The FSR considers six cardiovascular risk factors, including age (over than 20), gender (male and female), total cholesterol (TC), HDLcholesterol, systolic blood pressure, and smoking habits. This section is an interactive tool for users to calculate the heart attack risk score in every $\log$-in and obtain feedback via the text by the three traffic lights that are illustrated for three levels of risks (high, moderate and low risk). If the estimation of risk was over $20 \%$, red light will bright and for risk between 10 to $20 \%$ and less than 10\% orange and green light will turn on respectively. Users will receive an explanation regarding their score and will be guided to educational materials on the personal homepage.

5. Anthropometric and clinical measures: in this section user will record periodic measurements of weight, waist circumference, BMI and blood pressure, Total cholesterol, LDL-cholesterol, HDL-cholesterol, triglycerides, and Fasting blood glucose. These measurements will be displayed in a simple graph with three warning color (red $=$ needs attention, orange $=$ close to risk and green $=$ good) for each recording. The risk factors will draw at the sequence diagram automatically and will show level of risks for individual separately.

6. The questionnaires: users will complete a set of study questionnaires.

\section{Control}

We will use a waiting list as the control group. In fact all registrants will be exposed to the intervention but those who register late will be kept in waiting list and as indicated with be treated as control group for the study. Thus, participants who will be assigned to this group will receive an e-mail message every 3 weeks to keep them involved in the study during the intervention period but they will not have access to the My Healthy Heart Profile and they will not know that they are control group. The e-mail messages will contain general information about metabolic syndrome and healthy nutrition and benefits of fruit and vegetable intakes, physical activity and body weight loss. However, one should notice that such a control group in essence is an intervention group and not a true control group.

\section{Primary outcomes}

The primary outcomes are feasibility, acceptability, usability, and the change in metabolic syndrome components. Measures for assessing feasibility will include: the proportion of participants who respond to log-in to My Healthy Heart Profile for the first time, and response rate to postal follow-up for clinical assessments and complete the study questionnaires [77]. Acceptability will be explored by the number of sign-in to the My Healthy Heart Profile during intervention. Usability of the intervention will be accessed by the educational materials downloads and using of website components by participants. The change in metabolic syndrome components will be assessed by calculating changes in the following measures: waist circumferences, weight, blood pressure, triglycerides, HDL-cholesterol, and fasting blood glucose. Waist circumference will be measured in horizontal plane, midway between the lowest rib and the iliac crest with a measuring tape in centimeter [6]. The weight of individual dressed in light clothing without shoes will be measured at each time using a calibrated scale (Seca model 8811021658). Blood pressure will be measured with mercury sphygmomanometer twice in the same arm after the individual seated at rest 10-15 min. The systolic and diastolic measurement represents the mean of two readings. Blood sampling will be collected for measurements of total cholesterol, triglycerides, LDLcholesterol, HDL-cholesterol, and fasting blood glucose for all participants. Overnight fasting for $12-14 \mathrm{~h}$ is needed before blood sampling. Venous blood samples (5 ml) will be collected. Body Mass Index (BMI) will be measured by individual's weight divided by the square of the height [78].

\section{Secondary outcomes}

Secondary outcomes will include assessments of the followings:

a. Health-related quality of life (HRQOL) using the SF-36 (Iranian version). The SF-36 is a very popular measure of health related quality of life among the general population. The psychometric properties of the Iranian version of the questionnaire are well documented [79],

b. Physical activity using the International Physical Activity Questionnaire at last 7 days (IPAQ) [80], a well-validated questionnaire in Iran $[81,82]$;

c. Food frequency using the validated Iranian version of Food Frequency Questionnaire (FFQ) [83], and 
d. Psychological well-being using the Iranian version of 12-item General Health Questionnaire (GHQ-12) [84].

Measurements will be made at baseline (T0), three (T1) and six (T2) months follow-up. Food frequency questionnaire will be completed only on $\mathrm{T} 0$ and $\mathrm{T} 2$.

\section{Sample size}

The sample size was calculated based on one standard deviation decrease (2.5) [85] in waist circumference as one of the most important components of MS [86]. As such a study with a power of $90 \%$ at $5 \%$ significance level would need 60 participants in each arm. Giving that there might be an attrition risk, 80 participants per each group were sought.

\section{Attrition prevention}

In order to prevent attrition and encourage people to take part in the study we will use five strategies appropriate to participants. Firstly, free blood testing will be carried out for all participants at all three assessment times. Secondly, the test results will send through e-mail within 24 hours. We will request from users to log-in the personal profile and record the new values. Thirdly, e-mail reminders will send to users when they are inactive for 2 weeks. Fourthly, in order to keep up to date and enhance the website visiting, the new information will be placed on the site monthly. Finally, all recruitment processes will be online.

\section{Statistical analysis}

The characteristics of participants will be summarize as proportions or means with standard deviations. To test the study hypotheses t-tests for independent samples will be performed. Comparisons of proportions will be carried out using the Pearson's chi-square test without continuity correction. For all parameters $95 \%$ confidence intervals will be defined. Two-sided $\mathrm{p}$ values of less than 0.05 will be regarded as statistical significant. All analyses will be performed with the SPSS software version 18. Between group differences will be evaluated by intent-totreat analysis using the Generalized Linear Mixed Models (GLMM).

\section{Ethics}

The ethics committee of the Tehran University of Medical Sciences approved the study protocol. Written informed consent is obtained from all participants.

\section{Discussion}

The Internet is promising medium to offer lifestyle interventions [87]. Perhaps this study will help in refining the intervention for future research and practice. The results from this study, if successful, might help Iranians in several ways. In the first instance it will help to set up similar interventions. Secondly it will help to reach target populations as quickly as possible.

The study by Bosak et al. showed that web-based intervention to increase physical activity in people with metabolic syndrome appears feasible [22]. Recent evidence support that lifestyle interventions including physical activity and low-calorie diet might lead to at least $10 \%$ weight loss [88].

Various studies showed a positive association between log-in frequency and weight loss [44]. This approach may also be more persuasive and motivational, with the ability to promote rapid changes in participant's behavior, but remains to be tested.

\section{Abbreviations}

CVD: Cardiovascular diseases; WC: Waist circumference; BP: Blood pressure; BMI: Body mass index; RCT: Randomized controlled trial; QOL: Quality of life; FFQ: Food frequency questionnaire; IPAQ: International Physical Activity Questionnaire; GHQ: General health questionnaire; IBW: Ideal body weight; CBW: Corrected body weight; FSR: Framingham risk score.

\section{Competing interests}

The author declares that they have no competing interests.

\section{Authors' contribution}

$L$ is the main investigator, designed the study, will collect the data, and wrote the first draft. DS is the supervisor of the study and contributed to the writing process. MN will help in recruitment; KM helped in statistics; MAF will provide diet plan and carry out online nutritional counseling. AM is supervisor of the study, contributed to all aspect of the study and provided the final manuscript. All authors read and approved the paper.

\section{Acknowledgments}

This manuscript originated from a PhD thesis (240\2425) by Leila Jahangiry, Department of Health Education and Promotion, School of Public Health, Tehran University of Medical Sciences, Tehran, Iran. We acknowledge the contributions of Tehran Heart Center for providing facilities to the study. Finally we wish to thank the Iranian Institute for Health Sciences Research (ACECR) for their time and support for this research.

\section{Author details}

${ }^{1}$ Health Education and Health Promotion Department, School of Public Health, Tabriz University of Medical Sciences, Tabriz, Iran. ${ }^{2}$ Health Education and Health Promotion Department, School of Public Health, Tehran University of Medical Sciences, Tehran, Iran. ${ }^{3}$ Tehran Heart Center, Tehran University of Medical Sciences, Tehran, Iran. ${ }^{4}$ Department of Epidemiology and Biostatistics, School of Public Health, Tehran University of Medical Sciences, Tehran, Iran. ${ }^{5}$ Department of Community Nutrition, Faculty of Health and Nutrition, Tabriz University of Medical Sciences, Tabriz, Iran. ${ }^{6}$ Mental Health Research Group, Health Metrics Research Center, Iranian Institutes for Health Sciences Research, ACECR, Tehran, Iran.

Received: 10 June 2013 Accepted: 21 July 2014

Published: 24 July 2014

\section{References}

1. Ersen C, Maurice N, Niels P, Sandra V, Roderik K: Initiation of health-behavior change among employees participating in a web-based health risk assessment with tailored feedback. J Occup Med Toxicol 2011, 6:5.

2. Burke V, Beilin LJ, Cutt HE, Mansour J, Williams A, Mori TA: A lifestyle program for treated hypertensive improved health-related behaviors and cardiovascular risk factors, a randomized controlled trial. J Clin Epidemiol 2007, 60:133-141. 
3. Esmaillzadeh A, Mirmiran P, Azadbakht L, Etemadi A, Azizi F: High prevalence of the metabolic syndrome in Iranian adolescents. Obesity 2006, 14:377-382.

4. Eberly LE, Prineas R, Cohen JD, Vazquez G, Zhi X, Neaton JD, Kuller LH: Metabolic syndrome: risk factor distribution and 18-year mortality in the Multiple Risk Factor Intervention Trial. Diabetes Care 2006, 29:123-130.

5. Zambon S, Zanoni S, Romanato G, Chiara Corti M, Noale M, Sartori L, Musacchio E, Baggio G, Crepaldi G, Manzato E: Metabolic syndrome and all-cause and cardiovascular mortality in an Italian elderly population: the Progetto Veneto Anziani (Pro.V.A.) study. Diabetes Care 2009, 32:153-159.

6. Alberti $K G$, Zimmet $P$, Shaw J: The metabolic syndrome: a new worldwide definition. Diabet Med 2006, 23:469-480.

7. Cleeman J, Grundy S, Becker D, Clark L: Expert panel on detection, evaluation and treatment of high blood cholesterol in adults. Executive summary of the third report of the National Cholesterol Education Program (NCEP) Adult Treatment Panel (ATP III). Jama 2001, 285:2486-2497.

8. Ma X, Zhu S: Metabolic syndrome in the prevention of cardiovascular diseases and diabetes: still a matter of debate. Eur J Clin Nutr 2013, 67:518-521.

9. Beck-Nielsen $\mathrm{H}$ : Introduction and Definition of the Metabolic Syndrome. Wien: Springer-VerlagWien; 2013:1-6.

10. Murray CJ, Vos T, Lozano R, Naghavi M, Flaxman AD, Michaud C, Ezzati M, Shibuya K, Salomon JA, Abdalla S, Aboyans V, Abraham J, Ackerman I, Aggarwal R, Ahn SY, Ali MK, Alvarado M, Anderson HR, Anderson LM, Andrews KG, Atkinson C, Baddour LM, Bahalim AN, Barker-Collo S, Barrero LH, Bartels DH, Basáñez MG, Baxter A, Bell ML, Benjamin EJ, et al: Disability-adjusted life years (DALYs) for 291 diseases and injuries in 21 regions, 1990-2010: a systematic analysis for the global burden of disease study 2010. Lancet 2012, 380:2197-2223.

11. Mottillo S, Filion KB, Genest J, Joseph L, Pilote L, Poirier P, Rinfret S, Schiffrin EL, Eisenberg MJ: The Metabolic syndrome and cardiovascular risk: a systematic review and meta-analysis. J Am Coll Cardiol 2010, 56:1113-1132.

12. Eckel RH, Grundy SM, Zimmet PZ: The metabolic syndrome. Lancet 2005, 365:1415-1428.

13. Zabetian A, Hadaegh F, Sarbakhsh P, Azizi F: Weight change and incident metabolic syndrome in Iranian men and women; a 3-year follow-up study. BMC Public Health 2009, 9:138.

14. Azizi F, Hadaegh F, Khalili D, Esteghamati A, Hosseinpanah F, Delavari A, Larijani B, Mirmiran P, Zabetian A, Mehrabi Y: Appropriate definition of metabolic syndrome among Iranian adults: report of the Iranian National Committee of Obesity. Arch Iran Med 2010, 13:426-428.

15. Kirby MG: Cardiovascular disease prevention, screening and early detection in Turkey. Cardiology 2010, 115:294-296.

16. Hoekstra T, Beulens JW, van der Schouw YT: Cardiovascular disease prevention in women: impact of dietary interventions. Maturitas 2009, 63:20-27.

17. Sarrafzadegan N, Kelishadi R, Esmaillzadeh A, Mohammadifard N, Rabiei K, Roohafza H, Azadbakht L, Bahonar A, Sadri G, Amani A: Do lifestyle interventions work in developing countries? Findings from the Isfahan Healthy Heart Program in the Islamic Republic of Iran. Bull World Health Organ 2009, 87:39-50.

18. Azadbakht L, Mirmiran P, Esmaillzadeh A, Azizi T, Azizi F: Beneficial effects of a dietary approaches to stop hypertension eating plan on features of the metabolic syndrome. Diabetes Care 2005, 28:2823-2831.

19. Rubenfire M, Mollo L, Krishnan S, Finkel S, Weintraub M, Gracik T, Kohn D, Oral EA: The metabolic fitness program: lifestyle modification for the metabolic syndrome: using the resources of cardiac rehabilitation. Cardiopulm Rehabil Prev 2011, 31:282-289.

20. Manzoni GM, Pagnini F, Corti S, Molinari E, Castelnuovo G: Internet-based behavioral interventions for obesity: an updated systematic review. Clin Practice Epidemiol Mental Health 2011, 7:19.

21. Wadden TA, Foster GD: Behavioral treatment of obesity. Am Soc Clin Nutr 2005, 82(Suppl):230-235.

22. Bosak KA, Yates B, Pozehl B: Feasibility of an internet physical activity intervention. West J Nurs Res 2009, 31:648-661.

23. Riper H, Kramer J, Smit F, Conijn B, Schippers G, Cuijpers P: Web-based self-help for problem drinkers: a pragmatic randomized trial. Addiction 2008, 103:218-227.

24. Barak A, Proudfoot JG: Defining internet-supported therapeutic interventions. Ann Behav Med 2009, 38:4-17.
25. Hutton HE, Wilson LM, Apelberg BJ, Tang EA, Odelola O, Bass EB, Chander $\mathrm{G}$ : A systematic review of randomized controlled trials: Web-based interventions for smoking cessation among adolescents, college students, and adults. Nicotine Tob Res 2011, 13:227-238.

26. Bock B, Graham A, Sciamanna C, Krishnamoorthy J, Whiteley J, Carmona-Barros R, Niaura R, Abrams D: Smoking cessation treatment on the Internet: content, quality, and usability. Nicotine Tob Res 2004, 6:207-219.

27. Bock BC, Graham AL, Whiteley JA, Stoddard JL: A review of web-assisted tobacco interventions (WATIs). J Med Internet Res 2008, 10:39.

28. Walters ST, Wright JA, Shegog R: A review of computer and Internet-based interventions for smoking behavior. Addict Behav 2006, 31:264-277.

29. Shahab L, McEwen A: Online support for smoking cessation: a systematic review of the literature. Addiction 2009, 104:1792-1804.

30. Etter JF: The internet and the industrial revolution in smoking cessation counseling. Drug Alcohol Rev 2006, 25:79-84

31. Rooke S, Thorsteinsson E, Karpin A, Copeland J, Allsop D: Computer-delivered interventions for alcohol and tobacco use: a meta-analysis. Addiction 2010, 105:1381-1390.

32. Copeland J, Martin G: Web-based interventions for substance use disorders: a qualitative review. Subst Abuse Treat 2004, 26:109-116.

33. Finfgeld Connett D: Web-based treatment for problem drinking. Psychosoc Nurs Mental Health Serv 2006, 44:20-27.

34. Vernon ML: A review of computer-based alcohol problem services designed for the general public. Subst Abuse Treat 2010, 38:203-211.

35. White A, Kavanagh D, Stallman H, Klein B, Kay Lambkin F, Proudfoot J, Drennan J, Connor J, Baker A, Hines E: Online alcohol interventions: a systematic review. J Med Internet Res 2010, 12:62.

36. Cunningham JA, Khadjesari Z, Bewick BM, Riper H: Internet-based interventions for problem drinkers: From efficacy trials to implementation. Drug Alcohol Rev 2010, 29:617-622.

37. Bewick BM, Trusler K, Barkham M, Hill AJ, Cahill J, Mulhern B: The effectiveness of web-based interventions designed to decrease alcohol consumption: a systematic review. Prev Med 2008, 47:17-26.

38. Riper H, van Straten A, Keuken M, Smit F, Schippers G, Cuijpers P: Curbing problem drinking with personalized-feedback interventions: a meta-analysis. Am J Prev Med 2009, 36:247-255.

39. Carey KB, Scott-Sheldon LA, Elliott JC, Bolles JR, Carey MP: Computer-delivered interventions to reduce college student drinking: a meta-analysis. Addiction 2009, 104:1807-1819.

40. Tate DF: A series of studies examining internet treatment of obesity to inform internet interventions for substance use and misuse. Subst Use Misuse 2011, 46:57-65

41. Arem $\mathrm{H}$, Irwin M: A review of web-based weight loss interventions in adults. Obesity Rev 2011, 12:236-243.

42. Nguyen B, Kornman KP, Baur LA: A review of electronic interventions for prevention and treatment of overweight and obesity in young people. Obes Rev 2011, 12:298-314

43. Khaylis A, Yiaslas T, Bergstrom J, Gore-Felton C: A review of efficacious technology-based weight-loss interventions: five key components. Telemed J Health 2010, 16:931-938.

44. Neve M, Morgan PJ, Jones PR, Collins CE: Effectiveness of web-based interventions in achieving weight loss and weight loss maintenance in overweight and obese adults: a systematic review with meta-analysis. Obes Rev 2009, 11:306-321.

45. Azar M, Gabbay R: Web-based management of diabetes through glucose uploads: has the time come for telemedicine? Diabetes Res Clin Pract 2009, 83:9-17.

46. Ramadas A, Quek KF, Chan CK, Oldenburg B: Web-based interventions for the management of type 2 diabetes mellitus: a systematic review of recent evidence. Int J Med Inform 2011, 80:389-405.

47. Jackson CL, Bolen S, Brancati FL, Batts-Turner ML, Gary TL: A systematic review of Interactive computer assisted technology in diabetes care. Gen Intern Med 2006, 21:105-110.

48. Kaufman $\mathrm{N}$ : Internet and information technology use in treatment of diabetes. Int J Clin Pract 2010, 166(Suppl):41-46.

49. Robertson L, Smith M, Castle D, Tannenbaum D: Using the Internet to enhance the treatment of depression. Aust Psychiatry 2006, 14:413-417.

50. Wade AG: Use of the Internet to assist in the treatment of depression and anxiety: a systematic review. Prim Care Companion J Clin Psychiatry 2010, 12:09r00876. 
51. Van't Hof E, Cuijpers P, Stein DJ: Self-help and Internet-guided interventions in depression and anxiety disorders: a systematic review of meta-analyses. CNC Spectrums 2009, 14:34-40

52. Calear AL, Christensen $\mathrm{H}$ : Review of internet-based prevention and treatment programs for anxiety and depression in children and adolescents. Med J Aust 2010, 192(Suppl 11):S12-S14.

53. StjernswArd S, Astman M: Potential of e-health in relation to depression: short survey of previous research. Psychiatric Mental Health Nurs 2006, 13:698-703.

54. Spek V: Internet-based cognitive behavior therapy for symptoms of depression and anxiety: a meta-analysis. Psychol Med 2007, 37:319-328.

55. Barak A, Hen L, Boniel-Nissim M, Shapira N: A comprehensive review and a meta-analysis of the effectiveness of internet-based psychotherapeutic interventions. J Technol Hum Serv 2008, 26:109-160.

56. Mauriello LM, Sherman KJ, Driskell MM, Prochaska JM: Using interactive behavior change technology to intervene on physical activity and nutrition with adolescents. Adolesc Med State Art Rev 2007, 18:383-399.

57. Ferney SL, Marshall AL, Eakin EG, Owen N: Randomized trial of a neighborhood environment-focused physical activity website intervention. Prev Med 2009, 48:144-150.

58. Nakade M, Muto T, Hashimoto M, Haruyama Y: Internet-based education program of nutrition as a workplace health promotion tool: A review of the literature. Int Congr Ser 2006, 1294:135-138.

59. Brug J, Oenema A, Kroeze W, Raat H: The internet and nutrition education: challenges and opportunities. Eur J Clin Nutr 2005, 59(Suppl 1):S130-S137.

60. Murray E, Burns J, See TS, Lai R, Nazareth I: Interactive health communication applications for people with chronic disease. Cochrane Database Syst Rev 2005

61. Duncan MJ, Vandelanotte C, Rosenkranz RR, Caperchione CM, Ding H, Ellison M, George ES, Hooker C, Karunanithi M, Kolt GS, Maeder A, Noakes M, Tague R, Taylor P, Viljoen P, Mummery WK: Effectiveness of a website and mobile phone based physical activity and nutrition intervention for middle-aged males: trial protocol and baseline findings of the man up study. BMC Public Health 2012, 12:656.

62. Kraft P, Botelho R, Webb TL, Joseph J, Yardley L, Michie S: Using the Internet to Promote Health Behavior Change: A Systematic Review and Meta-analysis of the Impact of Theoretical Basis, Use of Behavior Change Techniques, and Mode of Delivery on Efficacy. J Med Intern Res 2010, 12:e4.

63. Schubart JR, Stuckey HL, Ganeshamoorthy A, Sciamanna CN: Chronic health conditions and internet behavioral interventions: a review of factors to enhance user engagement. Comput Inform Nurs 2011, 29:81-92.

64. Leslie E, Marshall AL, Owen N, Bauman A: Engagement and retention of participants in a physical activity website. Prev Med 2005, 40:54-59.

65. Verheijden MW, Jans MP, Hildebrandt VH, Hopman-Rock M: Rates and determinants of repeated participation in a web-based behavior change program for healthy body weight and healthy lifestyle. J Med Intern Res 2007, 9:e1

66. Fox MP: A systematic review of the literature reporting on studies that examined the impact of interactive, computer-based patient education programs. Patient Educ Couns 2009, 77:6-13.

67. Short CE, Vandelanotte C, Dixon MW, Rosenkranz R, Caperchione C, Hooker C, Karunanithi M, Kolt GS, Maeder A, Ding H, Taylor P, Duncan MJ: Examining participant engagement in an information technology-based physical activity and nutrition intervention for men: the manup randomized controlled trial. JMIR Res Protoc 2014, 3:e2.

68. Internet World Stats: Usage and Population Statistics. http://www internetworldstats.com/stats5.htm.

69. Rashidian A, Khosravi A, Khabiri R, Khodayari-Moez E, Elahi E, Arab M, Radaie Z: Islamic Republic of Iran's Multiple Indicator Demograpphic and Healh Survey (IrMIDHS) 2010. Tehran: Ministry of Health and Medical Education; 2012.

70. Delavari A, Forouzanfar MH, Alikhani S, Sharifian A, Kelishadi R: First nationwide study of the prevalence of the metabolic syndrome and optimal cutoff points of waist circumference in the Middle East: the national survey of risk factors for non communicable diseases of Iran. Diabetes Care 2009, 32:1092-1097.

71. Azizi F, Khalili D, Aghajani H, Esteghamati A, Hosseinpanah F, Delavari A, Larijani B, Kelishadi R, Hadaegh F: Appropriate waist circumference cut-off points among Iranian adults: the first report of the Iranian National Committee of Obesity. Arch Iran Med 2010, 13:243-244.
72. Esteghamati A, Abbasi M, Rashidi A, Meysamie A, Khalilzadeh O, Haghazali M, Asgari F, Nakhjavani M: Optimal waist circumference cut-offs for the diagnosis of metabolic syndrome in Iranian adults: results of the third national survey of risk factors of non-communicable diseases (SuRFNCD-2007). Diabet Med 2009, 26:745-746.

73. Simon S: A simple approach for randomization [electronic rapid response]. BMJ 1999, 319:703-704. 17 Sep. 1999. Responses to: Altman DG, Bland JM. Statistics notes: how to randomize.

74. Michie S, Richardson M, Johnston M, Abraham C, Francis J, Hardeman W Eccles MP, Cane J, Wood CE: The behavior change technique taxonomy (v1) of 93 hierarchically clustered techniques: building an international consensus for the reporting of behavior change interventions. Ann Behav Med 2013, 46:8-95.

75. National Institute of Health: Clinical Guidelines on the Identification, Education, and Treatment of Overweight and Obesity in Adults: The Evidence Report. USA: NIH; 1998

76. Hamwi GL: Changing dietary concepts in Diabetes Mellitus: Diagnosis and Treatment. New York: American Diabetes Association; 1964.

77. Jennings CA, Vandelanotte $C$, Caperchione CM, Mummery WK: Effectiveness of a web-based physical activity intervention for adults with Type 2 diabetes: a randomized controlled trial. Prev Med 2014, 60:33-40.

78. Global Database Body Mass Index. http://apps.who.int/bmi/index.jsp.

79. Montazeri A, Goshtasebi A, Vahdaninia M, Gandek B: The Short Form Health Survey (SF-36): translation and validation study of the Iranian version. Qual Life Res 2005, 14:875-882.

80. International Physical Activity Questionnaire: http://www.ipaq.ki.se/ipaq.htm.

81. Vasheghani-Farahani A, Tahmasbi M, Asheri H, Ashraf H, Nedjat S, Kordi R: The Persian, last 7-day, long form of the International Physical Activity. Questionnaire: translation and validation study. Asian J Sports Med 2011, 2:106-116.

82. Baghiani Moghaddam MH, Allahverdipour H, Dabagh Nikookheslat S, Safarpour S: The Iranian Version of International Physical Activity Questionnaire (IPAQ) in Iran: content and construct validity, factor structure, internal consistency and stability. World Applied Sci J 2012, 18:1073-1080

83. Hosseini Esfahani F, Asghari G, Mirmiran P, Azizi F: Reproducibility and relative validity of food group intake in a food frequency questionnaire developed for the Tehran Lipid and Glucose Study. J Epidemio/ 2010, 20:150-158.

84. Montazeri A, Harirchi AM, Shariati M, Garmaroudi G, Ebadi M, Fateh A: The 12-item General Health Questionnaire (GHQ-12): translation and validation study of the Iranian version. Health Q Life Outcomes 2003, 1:66

85. Bo S, Baldi C, Benini L, Dusio F, Forastiere G, Lucia C, Nuti C, Durazzo M, Cassader M, Gentile L: Effectiveness of a lifestyle intervention on metabolic syndrome: A randomized controlled trial. J Gen Intern Med 2007, 22:1695-1703.

86. Despres JP, Lemieux I: Abdominal obesity and metabolic syndrome. Nature 2006, 444:881-887.

87. Lustria MLA, Cortese J, Noar SM, Glueckauf RL: Computer-tailored health interventions delivered over the Web: review and analysis of key components. Patient Educ Couns 2009, 74:156-173.

88. Lundgren JD, Malcolm R, Binks M, O'Neil PM: Remission of metabolic syndrome following a 15-week low-calorie lifestyle change program for weight loss. Int J Obes 2008, 33:144-150.

doi:10.1186/1471-2458-14-748

Cite this article as: Jahangiry et al:: 'Red Ruby': an interactive web-based intervention for lifestyle modification on metabolic syndrome: a study protocol for a randomized controlled trial. BMC Public Health 2014 14:748. 